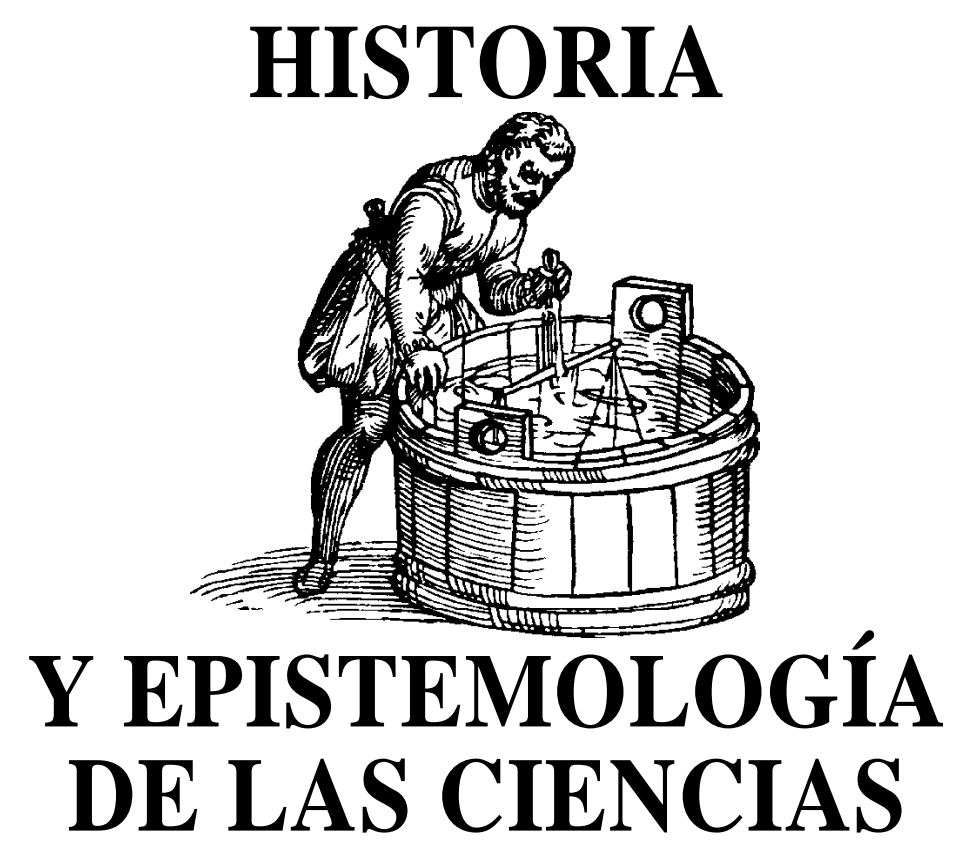

\title{
CARACTERÍSTICAS DEL PROCESO DE ENSEÑANZA-APRENDIZAJE DE LA FÍSICA EN LAS CONDICIONES CONTEMPORÁNEAS
}

VALDÉS CASTRO, PABLO y VALDÉS CASTRO, ROLANDO

Departamento de Física. ISP Enrique José Varona. 11400 Ciudad de la Habana. Cuba.

\section{SUMMARY}

The thesis that one of the fundamental objectives of science education is «to transmit» to the new generations the essential elements of scientific research experience acquired by mankind is argued. Certain aspects that, in opinion of the authors, should be considered in the physics teaching-learning process today are emphasized. One of the most outstanding characteristic of the contemporary scientific research activity is the use of computers; from this perspective the authors examine the basic objectives of their use in physics teaching.

\section{INTRODUCCIÓN}

Son de reconocimiento general las dificultades que confronta la enseñanza de la física en la escuela media, dificultades que con frecuencia se extienden a los primeros años de las carreras universitarias (Solbes, Calvo y 
Pomer, 1994; Oñorbe y Sánchez, 1996; Salinas, Cudmani y Pesa, 1996). En particular, es considerable el número de estudiantes que luego de la enseñanza recibida no domina los conceptos básicos, no adquiere las habilidades intelectuales que se esperaban o no manifiesta una actitud crítica durante el análisis de las cuestiones examinadas. A esta ya de por sí seria situación se suma la imperiosa necesidad de actualizar los cursos; urge la tarea de ponerlos en correspondencia con los adelantos de la ciencia y de la técnica, así como de reflejar mejor en ellos la enorme importancia que tiene la física para la vida de la sociedad.

La necesidad de actualizar los cursos de física ha sido subrayada por autores de populares libros de texto y por numerosos profesores e investigadores (Alonso 1994; Resnick, Halliday y Krane, 1994; Berazaín 1997; Patiño, Moreno y Sotto, 1997...). Es necesario tener presente, sin embargo, que esa actualización ha de abarcar tanto el sistema de conocimientos y las aplicaciones técnicas que se consideran en los cursos, hacia lo cual usualmente se dirige el interés principal, como las $c a$ racterísticas del propio proceso de enseñanza-aprendizaje, cuestión a la que se ha dedicado mucha menor atención. Así, mientras que en los cursos de física suelen incluirse -en mayor o menor medida, en dependencia del nivel de enseñanza de que se trate- conocimientos y aplicaciones técnicas correspondientes a las últimas décadas, los métodos y formas de trabajo empleados a menudo no reflejan ni tan siquiera los que se utilizaban en la ciencia hace ya más de tres siglos, en la época de Galileo y Newton.

La aproximación del aprendizaje de las ciencias a la actividad científico-investigadora -al menos en lo que se refiere a algunos elementos esenciales- aparece en nuestros días como una de las tendencias innovadoras más prometedoras para encarar la doble problemática señalada: $a$ ) las dificultades en la enseñanza, y $b$ ) la necesidad de relacionar a los estudiantes con métodos y formas de trabajo actualmente utilizados en la actividad científica. No obstante, esta concepción del aprendizaje necesita ser debidamente fundamentada, y la propia expresión actividad investigadora requiere ser concretada. En particular, debemos responder a dos preguntas básicas:

¿Por qué aprendizaje de la física como actividad científico-investigadora?

\section{¿De qué actividad investigadora se trata?}

Ahondar en la primera cuestión tiene suma importancia, si queremos que esta concepción del aprendizaje no se considere, como es frecuente, simplemente una alternativa para que los estudiantes adquieran conocimientos más profundos y sólidos y que ante todo se vea en ella una condición indispensable para relacionarlos con aspectos que forman parte esencial de la cultura de la sociedad contemporánea. Responder a la segunda cuestión tiene especial interés si se desea evitar que dicha orientación se identifique con corrientes innovadoras de los años sesenta (indudablemente progresistas para aquella época, pero cuyas limitaciones han sido hoy superadas por la investigación didáctica) y, además, para que la actividad científica tomada en consideración (cuerpo de conocimientos, medios técnicos, formas de trabajo y, en general, recursos utilizados) no se reduzca, como muchas veces ocurre, a la de siglos pasados.

A partir de determinadas posiciones teóricas y apoyándonos en el estudio bibliográfico y en nuestra propia experiencia en la enseñanza de la física, en el presente trabajo intentamos responder, en forma generalizada, a las dos interrogantes formuladas. Durante la elaboración de las respuestas fueron numerosos los aspectos considerados y, por consiguiente, algunos de ellos sólo pudieron ser tratados muy concisamente. Hemos procurado paliar esta limitación, incluyendo referencias a trabajos en los cuales dichos aspectos se analizan con mucha mayor amplitud.

Comenzaremos por la respuesta a la primera de las interrogantes, es decir, por fundamentar la necesidad de aproximar el aprendizaje de la física a la actividad científico-investigadora.

\section{FUNDAMENTOS SOCIOLÓGICOS, EPIS- TEMOLOGICOS Y PSICOLOGICOS DE LA CONCEPCIÓN DEL PROCESO DE APRENDIZAJE DE LA FÍSICA COMO ACTIVIDAD INVESTIGADORA}

Primero procuraremos mostrar que el énfasis que hoy se hace en la necesidad de aproximar el aprendizaje de las ciencias a la actividad científica está determinado, primordialmente, por razones sociales. Luego examinaremos otras razones de carácter psicológico y epistemológico, también de gran importancia para argumentar esta orientación del aprendizaje.

\section{Familiarización de los estudiantes con elementos de la experiencia investigadora: una obligación de la escuela contemporánea}

Fundamentar desde una perspectiva pedagogógicosocial la necesidad de aproximar el aprendizaje de la física a la actividad investigadora exige profundizar en los objetivos de la educación científica a la luz del concepto mismo de educación. Ya en el siglo pasado, el más sobresaliente de los forjadores de la cultura cubana, José Martí, precisó este concepto:

«Educar es depositar en cada hombre toda la obra humana que le ha antecedido: es hacer a cada hombre resumen del mundo viviente, hasta el día en que vive: es ponerlo a nivel de su tiempo, para que flote sobre él [...]» (Martí, 1975).

En 1926, Paul Langevin, al referirse al significado de la educación científica, decía (citado de acuerdo con Gil et al., 1991): «En reconocimiento del papel jugado por la ciencia en la liberación de los espíritus y en la afirmación 
de los derechos del hombre, el movimiento revolucionario hace un esfuerzo considerable para introducir la enseñanza de las ciencias en la cultura general y conformar esas humanidades modernas que aún no hemos logrado establecer.»

Por su parte, León Lederman, laureado con el Premio Nobel de Física, ha expresado (1984): «Dentro de la comunidad científica, sin embargo, la mayoría reconoce que el aspecto más importante de la ciencia es el cultural». Y más adelante continuaba: «[...] la sociedad tiene que preocuparse por la ciencia del mismo modo que se preocupa por otras actividades creativas suyas, tales como el arte, la música y la literatura».

Pudieran citarse las palabras de otras muchas personalidades que a lo largo de la historia se han referido al significado de la educación científica. Pero lo que aquí queremos resaltar es la idea de que el objetivo fundamental de la educación, y en particular de la enseñanza de las ciencias, es «transmitir» a las nuevas generaciones los elementos principales de la experiencia históricosocial acumulada, de la cultura de la humanidad (Danilov et al., 1978; Coll, 1987). Y estos elementos, cualquiera que sea la rama de la cultura de que se trate, pueden expresarse de modo generalizado en la forma siguiente (Danilov et al., 1978):

- Sistema de conocimientos sobre la naturaleza, la sociedad, los modos de actuar.

- Experiencia en la realización de acciones (plasmada en hábitos y habilidades) y, muy especialmente, en la actividad investigadora, creadora (expresada en la preparación para la solución de nuevos problemas).

- Determinada actitud hacia el mundo y el proceso de su conocimiento (expresada en el comportamiento y el sistema de valores de las personas).

Lo anterior indica que, pese a la excepcional importancia que tienen los conocimientos, debemos tener muy presente que éstos constituyen sólo uno de los componentes de la experiencia histórico-social. De aquí que el aprendizaje de la física, entendida ésta en su cabal acepción, como actividad cultural, supone la adquisición de ciertos conocimientos y habilidades, pero también de determinada experiencia en la actividad científico-investigadora, de actitudes y valores, con la particularidad de que cada uno de estos elementos debe estar actualizado «hasta el día en que vive» el estudiante.

Lamentablemente, a este enfoque de la educación científica, tradicionalmente se ha antepuesto la transmisión a los estudiantes de conocimientos ya preparados - a veces además demasiado específicos o desactualizados- y el desarrollo de habilidades particulares. En cambio, aspectos esenciales de la actividad científica, como el acotamiento de las situaciones consideradas, el planteamiento de hipótesis, la elaboración de estrategias para contrastarlas, etc., o el desarrollo en los estudiantes de cualidades del hombre de ciencia, como la perseverancia, la actitud crítica hacia la labor que realiza, la independencia, la iniciativa, etc., suelen no tenerse en cuenta.

La aspiración de aproximar el proceso de aprendizaje a la actividad investigadora constituye una tendencia progresista en la enseñanza de las ciencias desde hace ya varias décadas. Muchas de las propuestas realizadas reflejan, sin embargo, un enfoque inductivista o excesivas simplificaciones de lo que es el trabajo científico. Con frecuencia se ignora el papel central que el análisis cualitativo de la situación examinada, la formulación de hipótesis y, en general, todo el pensamiento divergente desempeñan en la actividad científico-investigadora, así como el carácter marcadamente social que en la actualidad tiene dicha actividad.

Hoy se advierte, por otra parte, un renovado interés hacia esta orientación del aprendizaje, lo que se explica por diversos factores:

- La creciente repercusión de la ciencia en la vida social y en la educación de las nuevas generaciones, el hecho de que se está originando un profundo cambio cultural con base en el desarrollo de la ciencia y la tecnología (Hurd, 1994) y, unido a esto, el importante papel que están desempeñando los medios de información en la divulgación de los adelantos científico-técnicos.

- Los cambios ocurridos en las características de la actividad científica en el objeto de estudio de las ciencias; en los medios de investigación, tanto intelectuales como técnicos; en la relación de la ciencia con la producción, etc.

- Una mejor comprensión de aspectos esenciales de la actividad científica y del proceso de enseñanza-aprendizaje, propiciada por el desarrollo de la filosofía, la psicología y la didáctica de las ciencias (Gil, 1993).

Y son esos mismos factores los que determinan que la estructuración del aprendizaje de la física como una actividad investigadora haya dejado hoy de ser una orientación simplemente conveniente, para convertirse en una necesidad impostergable.

Pero, como ya hemos señalado, existen además otras razones de carácter epistemológico y psicológico, que argumentan esta orientación del aprendizaje, las cuales examinamos a continuación.

Estructuración del proceso de enseñanza-aprendizaje de la física como actividad investigadora: vía idónea para la elaboración de conceptos, el desarrollo de una actitud científica y la motivación de los estudiantes por el aprendizaje

Es conocido que, luego de haber estudiado determinado tema durante varios cursos y en diferentes niveles de enseñanza, con frecuencia los estudiantes dan respuestas incorrectas a preguntas relativamente simples formuladas por el profesor. El análisis de esta situación ha dado lugar a una de las líneas de investigación en la 
didáctica de las ciencias en la que más se ha trabajado en las últimas décadas: la del estudio de los «errores conceptuales», «preconcepciones», etc. o, como más acertadamente se les ha denominado en los últimos años, concepciones alternativas.

Se han revelado concepciones alternativas de los estudiantes en relación con diferentes temas de física (Pfund y Duit, 1994). También se han descrito las características principales de dichas concepciones: coherencia interna; gran persistencia, dificultad para ser modificadas con estrategias de enseñanza convencionales; independencia del sexo, edad, e incluso a veces de la cultura; etc. (Wandersee, Mintzes y Novak, 1994; Pintó, Aliberas y Gómez, 1996). Se han reseñado, además, sus principales fuentes de procedencia: experiencias sensoriales, lenguaje habitualmente utilizado por los adultos o por los medios de comunicación, el propio proceso de enseñanza-aprendizaje, etc.

Algunos autores consideran que la conmoción originada por la evidencia que aportaban las investigaciones, de que los estudiantes luego de una enseñanza «rigurosa» no eran capaces de responder a «simples» preguntas, constituyó el impulso principal a las orientaciones constructivistas en el aprendizaje de las ciencias y, en particular, a la orientación de «cambio conceptual» (Gil et al., 1991).

Pero enfrentar con profundidad el problema de cómo reestructurar las concepciones de los estudiantes, de cómo transformarlas en científicas, requiere explicar el origen de tales concepciones. Y explicar dicho origen no consiste en señalar cuáles son esas concepciones en diferentes temas de la física, describir sus características o puntualizar sus posibles fuentes, por importante que todo esto sea. Lo principal es entender que, independientemente de su procedencia, todas ellas tienen un denominador común: representan un conocimiento de la apariencia de las cosas, un conocimiento superficial. Las concepciones científicas constituyen, por el contrario, un conocimiento en profundidad, más amplio e integral de dichas cosas.

Es necesario comprender que el ser humano es apto para conocer el mundo sólo así: primero ha de conocer lo dado de modo inmediato, la apariencia, y únicamente después le resulta posible revelar lo que está oculto, lo determinante. Si se tiene en cuenta esta regularidad del proceso del conocimiento, entonces no hay por qué asombrarse de la presencia, en los estudiantes, de ideas alternativas, «erróneas». Al iniciarse el tratamiento de determinado tema, éstas sencillamente reflejan una aún insuficiente profundidad en la comprensión de los conceptos estudiados. Lo verdaderamente preocupante debería ser: a) la persistencia de algunas de esas ideas luego de haber recibido varios años de educación científica en la escuela, e incluso en las universidades (por ejemplo, la idea de que se necesita aplicar determinada fuerza sobre un cuerpo para que se mueva; que, si se dejan caer dos piedras desde una misma altura, llegará primero al suelo la de mayor masa, etc.); y $b$ ) el surgimiento y asentamiento de muchas de ellas durante el propio proceso de enseñanza-aprendizaje (la identificación de la trayectoria de un cuerpo con el gráfico $x$ $t$ de su movimiento, la creencia de que la amplitud de las oscilaciones armónicas amortiguadas siempre decrece exponencialmente, etc.). Ambas circunstancias ponen de manifiesto la ineficacia del tipo de enseñanza que habitualmente practicamos. En este sentido debemos preguntarnos: ¿Cuál es el tipo de enseñanza que puede contribuir a superar las dificultades en el aprendizaje, en particular la presencia y persistencia de concepciones no científicas en los estudiantes?

Desde las posiciones de una concepción de la educación científica como la que hemos intentado esbozar en el apartado anterior, la respuesta no puede ser otra que un tipo de enseñanza que centre su atención en la orientación del aprendizaje como una actividad científicoinvestigadora (Gil, 1993). Y, en efecto, quizás el rasgo general que mejor caracteriza la investigación científica es, precisamente, el de ser una actividad orientada a profundizar a partir de la apariencia de las cosas, en lo que está oculto, en lo que no apreciamos directamente.

Es oportuno puntualizar que en este modo de concebir la investigación no resulta esencial la naturaleza de los objetos sobre los cuales se realizan las acciones. Estos pueden ser: objetos reales o sus representaciones sensoriales directas, como es usual durante el aprendizaje de los niños pequeños; el lenguaje, oral o escrito, natural o simbólico, como frecuentemente ocurre entre los científicos; o una combinación de ambas cosas, es decir, ideas expresadas mediante el lenguaje, que se acompañan de ejemplos y apoyos sensoriales concretos, como tiene lugar en la mayoría de los casos y, en particular, en el proceso de enseñanza-aprendizaje.

No cabe, pues, diferenciar los procesos de formación de conceptos y de asimilación de éstos (Ausubel, Novak y Hanesian, 1983), tomando como base la naturaleza del objeto sobre el cual se realizan las acciones. Lo decisivo para diferenciar los modos mediante los cuales se aprenden conceptos es el carácter que tiene la actividad que se realiza: si su objetivo consiste en revelar lo que se oculta tras la apariencia de las cosas, estableciendo nuevas propiedades y relaciones entre ellas, o, por el contrario, consiste en asociar formalmente determinado contenido a palabras y símbolos, en repetir ciertas formulaciones. En este sentido debemos subrayar que, contrariamente a lo que a veces se considera, el aprendizaje significativo por recepción, en el que, sobre la base del lenguaje y la comunicación, se profundiza en el objeto estudiado estableciéndose nuevas propiedades y relaciones entre las cosas, constituye parte inseparable del aprendizaje como investigación.

La importancia de organizar el aprendizaje como una investigación trasciende también a la esfera de las actitudes. La práctica de la investigación educa cualidades de la personalidad como la iniciativa, la disciplina, la tenacidad, etc. Ella forma además la convicción de que el conocimiento es, por una parte, un proceso ilimitado 
de aproximación a la realidad, y, por otra parte, un producto del intelecto, una creación humana, lo cual genera una actitud de cuestionamiento y crítica constantes de las situaciones analizadas y, al propio tiempo, de planteamiento de hipótesis y de elaboración de proyectos para enfrentar las dificultades y resolver los problemas surgidos. No es de extrañar por eso que se organicen conferencias internacionales para discutir los aspectos ideológico-sociales de la enseñanza de ciencias como la matemática, aparentemente desvinculada de estos aspectos (NECC, 1993).

Mencionemos en favor del aprendizaje como actividad investigadora, por último, el importante papel motivador que ésta desempeña. Es conocido que las personas que llevan a cabo una labor de este tipo-científicos, escritores, artistas, etc.- , como regla, viven satisfechas de su trabajo, e incluso en muchos casos dicha actividad se convierte en el sentido de sus vidas. Por su parte, ha sido ampliamente reconocido que el principal factor impulsor del aprendizaje reside en sus propias características (Leontiev 1981; Ausubel, Novak y Hanesian 1983; Hodson 1994): en el deseo de conocer y plantearse interrogantes, en el desafío que representa la solución de problemas, en la participación activa del aprendiz en las tareas planteadas, en el éxito obtenido durante la realización de éstas, etc.

De este modo, hemos llegado nuevamente, esta vez desde una perspectiva epistemológica y psicológica, a la necesidad de aproximar el aprendizaje de la física a una actividad investigadora.

Para concluir el breve análisis realizado de los aspectos que fundamentan esta concepción del aprendizaje, plantearemos, una vez más, la primera de las preguntas formuladas en la introducción y en apretada síntesis intentaremos resumir la respuesta:

- ¿Por qué aprendizaje de la física como actividad científico-investigadora?: a) Porque el objetivo fundamental de la educación es reproducir en las nuevas generaciones lo mejor de la experiencia histórico-social de la humanidad, uno de cuyos elementos principales es la experiencia de la actividad investigadora, creadora. b) Porque la actitud científico-investigadora ha adquirido en la actualidad especial relevancia, abarcando casi todas las esferas de la vida, convirtiéndose en uno de los elementos fundamentales del profundo cambio cultural que se está operando en la sociedad. c) Porque la actividad investigadora constituye la vía idónea para que los estudiantes profundicen en las cuestiones estudiadas y reestructuren las concepciones que poseen, así como para desarrollar en ellos una postura crítica durante el análisis de las situaciones consideradas y motivarlos por el aprendizaje.

Pero en el proceso docente, el aprendizaje no tiene lugar espontáneamente, muy por el contrario, precisa de una efectiva dirección por parte del profesor. Esto supone contestar detenidamente a la segunda pregunta formulada: ¿De qué actividad investigadora se trata?
CARACTERÍSTICAS DEL PROCESO DE ENSENANZA-APRENDIZAJE DE LA FISICA COMO ACTIVIDAD INVESTIGADORA

Responder a la pregunta anterior requiere tener en cuenta, por una parte, las características generales que posee la actividad investigadora en la ciencia y, por otra parte, la especificidad del proceso de aprendizaje en las condiciones docentes, la circunstancia de que se trata de un proceso en que la actividad de los estudiantes se ve facilitada gracias a la intervención del profesor y a la utilización de ciertos recursos didácticos. Comenzaremos por examinar la primera de estas cuestiones.

\section{Aspectos esenciales de la actividad científico- investigadora contemporánea}

Entre las características esenciales de la actividad científica que se destacan en la bibliografía relativa a enseñanza de las ciencias, y que se sugiere tener en cuenta en el proceso docente, están las siguientes: contextualización histórico-social de los problemas considerados; intenso y prolongado proceso de familiarización con la situación estudiada, en el que ésta es analizada desde múltiples perspectivas; acotamiento de la situación y formulación del problema o pregunta; simulación y reconstrucción en el plano ideal de la situación considerada; planteamiento de hipótesis y operativización de éstas, preferentemente en forma matemática; elaboración de estrategias para contrastar las hipótesis planteadas; formulación de nuevas preguntas y problemas derivados de la investigación que se realiza (Gil, Carrascosa, Furió y MartínezTorregrosa, 1991; Wenham, 1993; Marton, Fensham y Chaiklin, 1994; Mathews, 1994; Nersessian, 1995). Algunos de estos aspectos han estado presentes desde los albores de la ciencia; otros, como la reconstrucción de la situación en el plano teórico, la operativización de las hipótesis en forma matemática y el diseño de experimentos para contrastarlas, se hicieron particularmente evidentes sólo en la época de Galileo y de Newton (Mathews, 1994) y se consideran características propias de la ciencia moderna.

Sin embargo, durante el presente siglo, y en especial en las últimas décadas, han surgido, o se han puesto de manifiesto con mayor claridad, determinadas características de la actividad científica, que es imprescindible tener muy en cuenta si aspiramos a actualizar los métodos y formas de trabajo que empleamos en el proceso de enseñanza-aprendizaje de la física. Por eso, aun sin pretensiones de dar una respuesta acabada, es necesario preguntarse: ¿Cuáles son las características distintivas de la actividad científica contemporánea, especialmente en el campo de la física, que es necesario considerar al organizar la actividad investigadora de los estudiantes?

Una mirada a la evolución que ha tenido la física en este siglo, en particular en los últimos cincuenta años, así como a la repercusión que actualmente tiene en la vida 
de la sociedad, permite distinguir, entre otras, las siguientes características:

- Significativa modificación de su objeto de estudio: énfasis en la investigación de los objetos del micromundo y del megamundo y una nueva visión del mundo macroscópico.

- Papel cada vez más relevante de la teoría en el conocimiento de la realidad, clara delimitación de objetos ideales despojados de ropaje sensorial y, en consecuencia, acentuamiento del aspecto intelectual de las investigaciones.

- Interpenetración de la teoría y la actividad productiva, paso a primer plano de la función proyectiva de la ciencia, junto a sus funciones descriptiva y explicativa.

- Acelerado proceso de integración de las ciencias: en la solución de los problemas participan cada vez en mayor medida especialistas de diversos campos del conocimiento; además, como resultado de la confluencia de diferentes ramas del saber han surgido nuevas ciencias (ciencia de materiales, optoelectrónica, informática, etc.).

- Amplia utilización de dispositivos electrónicos, de computadoras y, en general, de la automatización, como importantes medios de investigación.

- Afirmación del carácter colectivo de la actividad científico-investigadora (ampliación de los colectivos de investigación y de los intercambios entre ellos, crecimiento del número de revistas especializadas y de eventos científicos, etc.).

- Enorme repercusión social de los resultados de la actividad científico-técnica (rápida aplicación en la práctica y comercialización de los resultados, colosales implicaciones medioambientales, difusión masiva de los adelantos científico-técnicos, etc.).

Lo anterior sugiere que, si verdaderamente se desean reflejar en el proceso de enseñanza-aprendizaje de la física elementos esenciales de la experiencia investigadora contemporánea, entonces no es suficiente que los estudiantes realicen actividades como el análisis y acotamiento de la situación estudiada, la formulación de hipótesis y la operativización de ellas en forma matemática, el diseño de experimentos, etc. Se requiere, además: cuidar de que una parte de las situaciones consideradas estén orientadas hacia aplicaciones actuales de la física en la técnica, en otras esferas de la ciencia, o que tengan una proyección social; elevar el papel que desempeña el elemento intelectual, teórico, durante el análisis y resolución de los problemas; utilizar dispositivos electrónicos, computadoras y, en general, la automatización como importantes herramientas para la solución de los problemas planteados; promover formas de trabajo que realcen el carácter colectivo, social, de la actividad científica; etc. La incorporación de tales elementos al proceso de enseñanza-aprendizaje de la física constituye, sin lugar a dudas, uno de los mayores retos que ha de enfrentar la didáctica de la física en nuestros días.

\section{La actividad investigadora en las condiciones docentes}

Comenzaremos subrayando que la actividad investigadora no puede ser identificada de un modo simplista con la realización de actividades de laboratorio, como muchas veces ocurre en la enseñanza de la física. ¿Cómo no considerar, por ejemplo, la lectura analítica, consciente, de diversos materiales, si en la ciencia la mayor parte del tiempo de los investigadores se emplea en semejante actividad? Por otra parte, sabemos muy bien que los trabajos de laboratorio pueden llevarse a cabo como meras manipulaciones de instrumentos y fórmulas, sin tener en cuenta elemento alguno de investigación.

En el apartado anterior mostramos que la actividad mediante la cual en la ciencia se profundiza en la situación estudiada tiene una estructura específica, se compone de determinados elementos: acotamiento de la situación, planteamiento y fundamentación de hipótesis, elaboración de estrategias para contrastar dichas hipótesis, etc. Esto significa que, si el objetivo de leer un libro o escuchar una exposición es contribuir a acotar determinada situación, a fundamentar cierta hipótesis, etc., entonces las actividades de leer y escuchar, por «receptivas» que parezcan, tienen un carácter investigativo; a su vez, si la realización de determinada práctica no responde a la solución de algún problema, a la necesidad de contrastar alguna hipótesis previamente formulada, etc., entonces dicha práctica no tendrá un carácter investigativo, por «activos» que se manifiesten los estudiantes. Este análisis llama la atención, una vez más, sobre el hecho de que el elemento central de la actividad investigadora es intelectual, ya se realicen las acciones externas sobre objetos reales, sus representaciones, o sobre el lenguaje.

Hemos subrayado anteriormente el papel esencial que en el proceso docente desempeña una efectiva dirección del aprendizaje por parte del profesor. Y un elemento básico para esta dirección es un sistema de tareas o «programa de actividades» (Gil y Valdés, 1996c) cuidadosamente elaborado, capaz de estimular y de orientar adecuadamente a los estudiantes. ¿Qué condiciones debe cumplir ese sistema de tareas?

El estudio de esta cuestión muestra que entre esas condiciones se encuentran las siguientes:

- Poseer un adecuado nivel de dificultad y tomar en cuenta la experiencia previa de los estudiantes: sus conocimientos, habilidades, motivaciones (Ausubel, Novak y Hanessian, 1983; Driver, 1986; Gil, Carrascosa, Furió y Martínez-Torregrosa, 1991).

- Formular las tareas en contextos que sean significativos, de interés para los estudiantes, de tal modo que la actividad que se realiza adquiera sentido para ellos (Leontiev, 1981; Duschl, 1995; Stinner 1995).

- Incitar a los alumnos a realizar aquellas acciones específicas mediante las cuales en la ciencia se profundiza en el objeto estudiado, es decir, las acciones propias 
de la actividad científico-investigadora (véase el apartado anterior).

- Contribuir a convertir las características esenciales de la situación considerada, en objeto directo de las acciones intelectuales de los estudiantes, ya que se tiene conciencia sólo de aquello que es objeto de dichas acciones (Leontiev, 1981; Talízina, 1988).

- Favorecer un elevado nivel de generalización (Rubinstein, 1966; Talízina, 1988; Nersessian, 1995), en particular, contribuir a que los alumnos prescindan de aquellos aspectos de las situaciones estudiadas que resultan irrelevantes para los conceptos en cuestión y a que, en cambio, retengan sus características esenciales y elaboren modelos genéricos de las situaciones.

Indudablemente que las características de la actividad que realizan los estudiantes y, en consecuencia, los resultados del aprendizaje, en gran medida, dependen de las tareas que se les plantean; en particular, un sistema de tareas que reúna las condiciones anteriores puede facilitar el aprendizaje de conceptos y contribuir a que los alumnos adquieran experiencia en importantes elementos de la actividad científica. Sin embargo, el sistema de tareas por sí mismo constituye sólo uno de los aspectos que determinan los resultados del aprendizaje; el otro aspecto es el propio proceso de interacción profesorestudiantes, en el cual se precisan o resaltan determinadas cuestiones, se proporcionan las ayudas pertinentes, se gradúa el nivel de independencia de los alumnos, se evalúa el aprendizaje, etc. Por consiguiente, pudiéramos ahora preguntarnos: ¿Qué otras condiciones, además de un sistema de tareas convenientemente preparado, es necesario tener en cuenta para dirigir eficazmente el proceso de aprendizaje de los estudiantes?

Entre ellas destacan las siguientes (las cuales, reiteramos, no están necesariamente implícitas en el sistema de tareas):

- Utilizar esquemas y modelos como vía para exteriorizar las acciones que se llevan a cabo en el plano ideal y como apoyo sensorial durante la experimentación mental con las situaciones analizadas. En los esquemas y modelos se plasman -y muchas veces incluso se acentúan- las características esenciales de la situación, al tiempo que se prescinde de los factores secundarios que intervienen en ésta, lo cual contribuye a elevar el grado de abstracción y generalización durante la solución de las tareas; además, la exteriorización de las acciones intelectuales permite un mejor control de ellas, tanto por parte del profesor como de los propios estudiantes (Talízina, 1988; Hennessy et al., 1995a).

- Favorecer una elevada independencia intelectual de los estudiantes durante la realización de las tareas (Talízina, 1988). El profesor no puede aprender por los estudiantes ni realizar las operaciones intelectuales por éstos, el trabajo de articular las nuevas ideas con la estructura de conocimientos que poseen sólo pueden realizarlo ellos mismos (Ausubel, Novak y Hanesian, 1983). Para que profundicen en lo estudiado e interiori- cen los conceptos científicos, es preciso, pues, que, aunque bajo la dirección del profesor, desplieguen una intensa actividad intelectual (acotamiento de la situación analizada y precisión del problema, de experimentación mental con la situación, planteamiento de hipótesis, formulación de nuevas preguntas, etc.), en la cual amplían y reestructuran sus conocimientos, elevándolos a niveles superiores de abstracción y generalización.

- Organizar el trabajo de los estudiantes en equipos (Wandersee, Mintzes y Novak, 1994; Hennessy et al., 1995a) y presentar y discutir en el colectivo los resultados obtenidos (Duschl, 1995). Esto permite exteriorizar, argumentar y debatir diversas ideas desde múltiples perspectivas, lo que esclarece el sentido de la tarea que se lleva a cabo y estimula la actividad intelectual de los estudiantes. Por otra parte, semejante proceder refleja formas de trabajo hoy ampliamente utilizadas en la actividad científica.

- Evaluar continuamente la actividad que se realiza. La evaluación constituye un aspecto esencial de la dirección de cualquier proceso y, en particular, de la dirección del aprendizaje. Señalemos, ante todo, que, para que sirva de estímulo al aprendizaje, ha de ser percibida por los estudiantes como una ayuda efectiva y generar en ellos expectativas positivas. Diversos investigadores coinciden en que la valoración de la propia actividad a lo largo de todo el proceso es más eficaz que la valoración de sus resultados terminales, aun cuando esta última se realice de modo frecuente (Talízina, 1988; Gil, Carrascosa, Furió y Martínez-Torregrosa, 1991). Desde la perspectiva de una concepción del aprendizaje como la que hemos estado desarrollando, debe constituir objeto de evaluación, en primer lugar, el trabajo realizado por los diferentes equipos durante la clase: tanto en lo que se refiere a sus aspectos conceptuales como a los metodológicos y actitudinales. Ello permite al profesor incidir positivamente a tiempo en el aprendizaje y posibilita a los estudiantes participar en la regulación de su propia actividad, al darles la oportunidad de valorar sus avances, rectificar los errores, evaluar su implicación en la realización de las tareas planteadas, etc.

En el proceso de enseñanza-aprendizaje de la física los estudiantes tienen la posibilidad de desarrollar un intenso trabajo intelectual, en particular, durante la resolución de cuestiones de lápiz y papel y las prácticas de laboratorio. Son éstas, actividades propicias para precisar los rasgos esenciales de los conceptos en elaboración y para que los estudiantes pongan de manifiesto las concepciones alternativas, no científicas, que poseen, lo que da la oportunidad al profesor de incidir sobre ellas; a través de dichas actividades los estudiantes tienen también la ocasión de practicar importantes aspectos de la actividad científico-investigadora. La resolución de problemas de «lápiz y papel» y la realización de prácticas de laboratorio con esta orientación han sido analizadas e ilustradas mediante ejemplos concretos en otros trabajos (Gil y Valdés, 1995a, 1996a, 1996b).

Finalmente debemos tener en cuenta que en nuestros días no es posible responder plenamente a la pregunta 
¿De qué actividad investigadora se trata?, sin considerar, como ya indicábamos, ciertas características de la actividad científica contemporánea. Entre estas características sobresale en la actualidad la utilización de las computadoras, lo que, por otra parte, constituye una de las tendencias innovadoras más extendidas en el proceso de enseñanza-aprendizaje en la última década. Dedicamos, pues, el próximo apartado a examinar brevemente esta cuestión.

\section{Utilización de las computadoras en el proceso de enseñanza-aprendizaje de la física}

El empleo de las computadoras en la enseñanza de la ciencias y, en particular de la física, es ya una realidad. Así lo evidencian publicaciones en diversas revistas (Wilson y Redish, 1989; Kocher, 1992; Field, 1995; Hasson y Bug, 1995; Herrán y Alonso, 1995, etc.; para una relación de revistas más extensa véase, por ejemplo, Berger et al., 1994), en comunicaciones presentadas en congresos y talleres (Gea et al., 1995, Carrasco y Seba1los, 1997; Lavagna et al., 1997; Quiles et al., 1997...), manuales de experimentos (Gallego, Lowy y Robles, 1991; Barrio et al., 1992...) y en populares libros de texto (Resnick, Halliday y Krane, 1994). Hoy las discusiones se centran alrededor de los objetivos que debe tener la introducción de esta tecnología. ¿Cuáles deben ser los objetivos fundamentales de la utilización de las computadoras en la enseñanza de la física?

En relación con esta cuestión pueden distinguirse dos orientaciones principales, las cuales reflejan dos aspectos esenciales -ya mencionados- que es necesario tener en cuenta al organizar el proceso de enseñanza-aprendizaje de las ciencias: las características que posee la actividad científica y la especificidad del proceso de aprendizaje en las condiciones docentes. En una de estas orientaciones, el objetivo fundamental de introducir las computadoras consiste en relacionar a los estudiantes con conceptos y procedimientos que caracterizan la actividad científica contemporánea (Valdés y Valdés, 1994; Herrán y Alonso, 1995; Rubayo y González, 1997). La otra orientación concibe la computadora, primordialmente, como un facilitador del aprendizaje, un medio de ayuda al profesor: tutorial interactivo, sistema multimedia, simulación de fenómenos, etc. (Barberá y Sanjosé, 1990; Abreu, Fábrega y Olivero, 1991; González, 1996; López, Montoya y Pancorbo, 1997). La mayoría de los trabajos se inscribe en esta segunda dirección o en la descripción de distintos aspectos técnicos u operativos del uso de las computadoras.

Así, muchas de las propuestas didácticas de simulación por computadoras tienen una función meramente ilustrativa o suponen que los estudiantes trabajan con modelos ya preparados, modelos en los que, lamentablemente, a veces se plasman sólo las características externas, perceptibles, del objeto estudiado. En este sentido es necesario tener muy en cuenta que en la ciencia la simulación se inicia, precisamente, con el proceso de elaboración del modelo y, además, que en éste se fijan las características esenciales, por lo general no tangibles, del objeto investigado.

Por su parte, en los manuales o instrucciones para la realización de experimentos docentes automatizados mediante computadoras (Gallego, Lowy y Robles, 1991; Barrio et al., 1992; González, 1996), casi nunca se prevé que los estudiantes participen en el diseño de ellos, olvidándose que el sentido de la automatización no es sólo elevar la eficacia en el registro y procesamiento de la información - por importante que ello sea- sino también liberarnos de la realización de ciertas operaciones elementales en beneficio de otras intelectuales de un nivel superior, tales como concebir la situación experimental, operar con ella en el plano mental evaluando la posibilidad de obtener los efectos previstos, proyectar la forma concreta en que se realizarán las mediciones y se procesarán los datos, etc.

En los últimos años se han elaborado valiosos programas informáticos que potencian la orientación investigadora en el aprendizaje de la física, pues dan la posibilidad a los estudiantes de representar esquemáticamente en la computadora determinada situación previamente imaginada, transformarla, variar los parámetros que la caracterizan, etc.; en otras palabras, les dan la oportunidad de participar en la construcción de modelos de la situación estudiada y en la experimentación con ellos (Hennessy et al., 1995a, 1995b). Tales representaciones esquemáticas en la computadora son similares a las que pueden hacerse mediante lápiz y papel y, como éstas, constituyen apoyos externos, visuales, durante el proceso de razonamiento y contribuyen a hacer abstracción de multitud de aspectos secundarios presentes en cualquier situación real, todo lo cual orienta el proceso del pensamiento. Pero el trabajo con estos programas informáticos tiene, respecto a la tradicional esquematización mediante lápiz y papel, la enorme ventaja de la inmediata retroalimentación: los resultados del razonamiento pueden contrastarse casi instantáneamente con los que ofrece la computadora en forma de esquema dinámico, de gráfico, o numérica. Esto permite a los alumnos diseñar y llevar a cabo sus propios experimentos, introducir modificaciones en los diseños o en el curso de los razonamientos, etc.

No obstante el gran valor didáctico de tales programas informáticos, cuando los estudiantes trabajan con ellos, generalmente no utilizan la computadora para los mismos fines con que se emplea en la ciencia: resolver problemas que sería difícil, o imposible, solucionarlos de otro modo. El objetivo principal de las simulaciones que en este caso realizan los estudiantes es favorecer el cambio conceptual (Hennessy et al., 1995a).

Desde la perspectiva de una enseñanza de la física que pretenda reflejar las características esenciales de la actividad científico-investigadora contemporánea, no es posible concebir las computadoras simplemente como un medio facilitador del proceso de aprendizaje, es necesario asignarles el lugar que por su origen y desarrollo les corresponde en la historia de la ciencia: poderosa herramienta para la solución de auténticos problemas. 
En consecuencia, debemos preguntarnos: ¿Cuáles son las direcciones principales de utilización de las computadoras en la ciencia que deben encontrar reflejo en la enseñanza de la física?

Un análisis histórico de la evolución de las computadoras y de las funciones que han desempeñado en la actividad científica pone de manifiesto que sus diferentes generaciones se han caracterizado no sólo por determinado desarrollo de la base técnica empleada y el mejoramiento de los diseños, sino también por importantes transformaciones metodológicas originadas en dicha actividad (Valdés, 1996). Tal análisis permite concluir, además, que estas transformaciones han estado relacionadas con el empleo de las computadoras en las siguientes direcciones:

- La resolución de problemas de cálculos numéricos durante la resolución de problemas.

- La realización de experimentos con modelos.

- El empleo de sistemas expertos.

- La automatización de experimentos.

En el marco de una concepción del proceso de enseñanza -aprendizaje de la física como la que hemos pretendido argumentar a lo largo del presente trabajo, son, pues, ésas, las direcciones que definen el campo fundamental de la actividad de los estudiantes con las computadoras. Esta orientación ha sido ilustrada mediante ejemplos concretos en otros trabajos (Gil y Valdés, 1995a, 1995b; Valdés, Valdés y Clavero, 1997).

\section{CONCLUSIÓN}

A modo de conclusión resumiremos los aspectos principales que hemos intentado destacar. Ante todo, la idea clave de que la concepción del aprendizaje como inves-

\section{REFERENCIAS BIBLIOGRÁFICAS}

ABREU, J., FÁBREGA, A. y OLIVERO, M. (1991). DILuz. Madrid: MEC.

ALONSO, M. (1994). Guest comment: Physics teachers are more conservative than the conservation laws. Am. J. Phys, 62(1), pp. 13-14

AUSUBEL, D., NOVAK, J. y HANESIAN,H.(1983). Psicología educativa. Un punto de vista cognoscitivo. México: Trillas.

BARRIO, C. et al. (1992). El ordenador en el laboratorio. 46 ejemplos prácticos para la enseñanza obligatoria. Madrid: PNTIC-MEC. tigación tiene su fundamento primero en la necesidad de «transmitir» a las nuevas generaciones la experiencia histórico-social, cultural, de la humanidad, uno de cuyos componentes esenciales es la experiencia en la actividad investigadora. En segundo lugar, la tesis de que el origen de las concepciones alternativas, no científicas, de los estudiantes es un conocimiento superficial de las cosas, de la apariencia de ellas, y que, en consecuencia, la vía idónea para superarlas consiste en profundizar en dichas cosas por medio de una actividad investigadora. En tercer lugar, el convencimiento de que la actividad investigadora no sólo contribuye a que los estudiantes alcancen niveles superiores de desarrollo cognoscitivo, sino también a formar en ellos valiosas cualidades de la personalidad y a motivarlos por el aprendizaje. En cuarto lugar es preciso insistir en que, al hablar de actividad investigadora en la enseñanza de la física deben tenerse presente, ante todo, las características esenciales de la actividad científica contemporánea: los medios técnicos e intelectuales, las formas de trabajo, etc., actualmente utilizados y, muy en particular, la utilización de las computadoras como herramienta fundamental para el análisis y solución de problemas. Naturalmente, también es necesario tomar en consideración las especificidad del proceso de aprendizaje en las condiciones docentes.

Digamos, para finalizar, que las ideas expuestas en este trabajo constituyen la base sobre la cual se apoyan las transformaciones que desde el curso 1995-96 se están llevando a cabo en el ciclo básico de física que se imparte en el Instituto Superior Pedagógico Enrique José Varona de la Habana. Los resultados ya obtenidos confirman las ventajas que, en la formación inicial de profesores de física tiene la aproximación del proceso de aprendizaje a una actividad investigadora: la profundidad en los conocimientos que adquieren los estudiantes es mayor, obtienen cierta práctica en elementos esenciales del trabajo científico. Pero, lo que desde nuestro punto de vista es más importante aún: la actitud de ellos hacia el aprendizaje, hacia la ciencia y la enseñanza de la ciencia, se torna cada vez más positiva.
BARBERÁ, Ó. y SANJOSÉ, V., 1990. Juegos de simulación por ordenador: un útil para la enseñanza a todos los niveles, Enseñanza de las Ciencias, 8(1), pp. 46-51.

BERAZAÍN, A. (1997). Actualización del curso de física general mediante la resolución de problemas, en Primer TallerIberoamericano de Enseñanza de la Física Universitaria. Libro de resúmenes. La Habana: Universidad de la Habana.

BERGER, C., LU, C., BELZER, S. y VOSS, B. (1994). Research on the Uses of Techonology in Science Education, en Gabel, D.L. (ed.). Handbook of Research on Science Teaching and Learnig. Nueva York: Macmillan Pub. Co. 
CARRASCO, B. y SEBALLOS, S. (1997). El computador en el laboratorio. Evaluación de una experiencia de cambio, en Primer Taller Iberoamericano de Enseñanza de la Física Universitaria. Libro de resúmenes. La Habana: Universidad de la Habana.

COLL, C. (1987). Psicología y curriculum. Barcelona: Editorial Laia.

DANILOV, M., SKATKIN, M., BUDARNI, A., SHAJMAIEV, N. y KRAIEVSKI, V. (1978). Didáctica de la escuela media. La Habana: Libros para la Educación.

DRIVER, R. (1986). Psicología cognoscitiva y esquemas conceptuales de los alumnos. Enseñanza de las Ciencias, 4 (1), pp. 3-15.

DUSCHL, R. (1995). Más allá del conocimiento: los desafíos epistemológicos y sociales de la enseñanza mediante el cambio conceptual. Enseñanza de las Ciencias, 13 (1), pp. 314.

FIELD, R. (1995). A spreadsheet simulation for a Young's double slits experiment. Physics Education, 30(4), pp. 230235 .

GALLEGO, A., LOWY, E. y ROBLES, J. (1991). Laboratorio asistido por ordenador. Madrid: PHYWE ESPAÑA.

GEA, M., HOYOS D., JAVI, V., POCOVÍ, C., ALURALDE, E., CADENA, C., GRAMAJO, C.,LOZANO,R.,PASSAMAI, V. y SARAVIA, L. (1995). Desarrollo de un sistema computarizado para experiencias de cinemática. Novena Reunión Nacional de Educación en la Física. Argentina: APFA.

GIL, D. (1993). Contribución de la historia y de la filosofía de las ciencias al desarrollo de un modelo de enseñanza-aprendizaje como investigación. Enseñanza de las Ciencias, 11(2), pp. 197-212.

GIL, D., CARRASCOSA, J., FURIÓ, C. y MARTÍNEZTORREGROSA, J. (1991). La enseñanza de las ciencias en la educación secundaria. Barcelona: Horsori.

GIL, D. y VALDÉS, P. (1995a). Un ejemplo de práctica de laboratorio como actividad investigadora. Alambique. Didáctica de las Ciencias Experimentales, 6, pp. 93-102.

GIL, D. y VALDÉS, P. (1995b). Contra la distinción clásica entre «teoría», prácticas experimentales» y «resolución de problemas»: el estudio de las fuerzas elásticas como ejemplo ilustrativo. Didáctica de las Ciencias (Experimentales y Sociales), 9, pp. 3-25.

GIL, D. y VALDÉS, P. (1996a). La orientación de las prácticas de laboratorio como investigación: un ejemplo ilustrativo. Enseñanza de las Ciencias, 14(2), pp. 155-163.

GIL, D. y VALDÉS, P. (1996b). La resolución de problemas de física: de los ejercicios de aplicación al tratamiento de situaciones problemáticas, en Cruz, A. et al. Temas escogidos de la didáctica de la física. La Habana: Pueblo y Educación.

GIL, D. y VALDÉS, P. (1996c). Tendencias actuales en la enseñanza-aprendizaje de la física, en Cruz, A. et al. Temas escogidos de la didáctica de la física. La Habana: Pueblo y Educación.

GALLEGO, A., LOWY, E. y ROBLES, J. (1991): Laboratorio asistido por ordenador. Madrid: PHYWE ESPAÑA.
GONZÁLEZ, O. (1996). Vías para activar el proceso docente en física general: una alternativa para el ase guramiento del nivel de partida y un modo de uso del ordenador en el estudio de situaciones experimentales. Tesis de doctorado. La Habana: Instituto Central de Ciencias Pedagógicas.

HASSON, B. y BUG, A. (1995). Hands-On and Computer Simulations. The Physics Teacher, 33(4), pp. 230-236.

HENNESSY, S., TWIGGER, D., DRIVER, R., O'SHEA, T., O'MALLEY, C., BYARD, M., DRAPER, S., HARTLEY, R., MOHAMED, R. y SCANLON, E. (1995a). Design of a computer-augmented curriculum for mechanics. International Journal of Science Education, 1(17), pp. 75-92.

HENNESSY, S., TWIGGER, D., DRIVER, R., O'SHEA, T., O'MALLEY, C., BYARD, M., DRAPER, S., HARTLEY, R., MOHAMED, R. y SCANLON, E. (1995b). A classroom intervention using a computer-augmented curriculum for mechanics. International Journal of Science Education. 2(17), pp. 189-206.

HERRÁN, C. y ALONSO, A. (1995). La utilización didáctica de la hoja de cálculo. Revista Española de Física, 9 (3), pp. 49-54.

HODSON, D. (1994). Hacia un enfoque más crítico del trabajo de laboratorio. Enseñanza de las Ciencias, 12(3), pp. 299-313.

HURD, P. (1994). New Minds for a New Age: Prologue to Modernizing the Science Curriculum. Science Education, 78 (1), pp. 103-116.

KOCHER, C. (1992). A laboratory course in computer interfacing and instrumentation. American Journal of Physics, 60(3), pp. 246-251.

LAVAGNA, M., BAADE, N., ZERBINO, L. y RÉBORA, G., (1997). Simulación por computadora y experimentación complementando la enseñanza de conceptos básicos de la óptica ondulatoria, en Primer Taller Iberoamericano de Enseñanza de la Física Universitaria. Libro de resúmenes La Habana: Universidad de la Habana.

LEDERMAN, L. (1984). The Value of Fundamental Science. Scientific American, 251 (5), pp. 34-41.

LEONTIEV, A. Cuestiones psicológicas de la teoría de la conciencia, en Actividad, conciencia, personalidad. La Habana: Pueblo y Educación.

LÓPEZ, V., MONTOYA, M., y PANCORBO, M. (1997). Práctica de física aplicando multimedia, en Primer Taller Iberoamericano de Enseñanza de la Física Universitaria. Libro de resúmenes. La Habana: Universidad de la Habana.

MANRIQUE, M.J., VARELA, P. y FAVIERES, A. (1989). Selección bibliográfica sobre esquemas alternativos de los estudiantes en electricidad. Enseñanza de las Ciencias, 7(3), pp. 292-295.

MARTÍ, J. (1975). Obras completas. Tomo 8. La Habana: Ciencias Sociales.

MARTON, F., FENSHAM, M. y CHAIKLIN, S. (1994). A Nobel's eye view of scientific intuition: discussions with the Nobel prize-winners in physics, chemistry and medicine (1970-86). International Journal of Science Educacion, 16 (4), pp. 457-473. 
MATHEWS, M. (1994). Historia, filosofía y enseñanza de las ciencias: la aproximación actual. Enseñanza de las Ciencias, 12(2), pp. 255-277.

NECC. Mathematics Commission (1993).Political dimensions of Mathematics Education 2. Caps Town: Maskew Miller Logman.

NERSESSIAN, J. (1995). Should Physicists Preach What they Practice? Constructive Modeling in Doing and Learning Physics. Science \& Education, 4, pp. 203-226.

OÑORBE, A. y SÁNCHEZ, J. (1996). Dificultades en la enseñanza-aprendizaje de los problemas de física y química. II. Opiniones del profesor. Enseñanza de las Ciencias, 14(3), pp. 251-260.

PATIÑO, A., MORENO, Y. y SOTTO, A. (1997). La modernización, actualización y especialización de un curso de física, en Primer Taller Iberoamericano de Enseñanza de la Física Universitaria. Libro de resúmenes. La Habana: Universidad de La Habana.

PFUNDT, H. y DUIT, R. (1994). Bibliography: Student's alternative frameworks and science education (4a. ed.). Kiel: Institute for Science Education at the University of Kiel.

PINTÓ, R., ALIBEROS, J. y GÓMEZ, R. (1996). Tres enfoques de la investigación sobre concepciones alternativas. Enseñanza de las Ciencias, 14(2), pp. 221-232.

QUILES, F., BENAVIDES, J., VILLATORO, F., MONTIJANO, M., SÁEZ, E., HERNÁNDEZ, M. y MOLINA, M. (1997). Automatización versus simulación de experimentos con computadores personales, en Primer Taller Iberoamericano de Enseñanza de la Física Universitaria. Libro de resúmenes. La Habana: Universidad de la Habana.

RESNICK, R., HALLIDAY, D. y KRANE, K. (1994). Física. Prefacio al Volumen 1. México: CECSA.

RUBAYO, J. y GONZÁLEZ, C. (1997). Empleo de los métodos numéricos en el cálculo de funciones de onda y niveles de energía de sistemas cuánticos unidimensionales, en Primer TallerIberoamericano de Enseñanza de la Física Universitaria. Libro de resúmenes. La Habana: Universidad de la Habana.
RUBINSTEIN, S. (1966). El proceso del pensamiento. La Habana: Universitaria.

SALINAS, J., CUDMANI, L. y PESA, M. (1996). Modos espontáneos de razonar: un análisis de su incidencia sobre el aprendizaje del conocimiento científico a nivel universitario básico. Enseñanza de las Ciencias, 14(2), pp. 209-220.

SOLBES, J., CALVO, A. y POMER, F. (1994). El futuro de la enseñanza de la física. Revista Española de Física, 8(4), pp. 45-49.

STINNER, A. (1995). Contextual Settings, Science Stories and Large Context Problems: Toward a More Humanistic Science Education. Science Education, 79(5), pp. 555-581.

TALIZINA, N. (1988). Psicología de la enseñanza. Moscú: Progreso.

VALDÉS, R. (1996). Objetivos fundamentales y metodología de la utilización de las computadoras en la enseñanza de la física. Consideraciones acerca de la formación y superiación de profesores. Tesis doctoral. La Habana. Departamento de Física, ISP Enrique J. Varona.

VALDÉS, R. y VALDÉS, P. (1994).Utilización de los ordenadores en la enseñanza de la física. Revista Española de Física, 8 (4), pp. 50-52.

VALDÉS, R., VALDÉS, P. y CLAVERO, A. (1997). Familiarización de los estudiantes con la automatización de experimentos mediante computadoras: determinación de la velocidad del sonido en le aire. (Aceptado para su publicación en Revista Española de Física.)

WANDERSEE, J., MINTZES, J. y NOVAK, J. (1994). Research on alternative conceptions in science, en Gabel, D. L. (ed.). Handbook of Research on Science Teaching and Learning Nueva York: Macmillan Pub. Co.

WENHAM, M. (1993). The nature and role of hypotheses in school science investigations. International Journal of Science Education, 15(3), pp. 231-240.

WILSON, J. y REDISH, E. (1989). Using computers in teaching physics. Physics Today, pp. 42-34. 